\title{
Spatial solitons in a medium composed of self-focusing and self-defocusing layers
}

\author{
Javid Atai ${ }^{1}$ and Boris A. Malomed ${ }^{2}$ \\ (1) School of Electrical and Information Engineering, University of Sydney, NSW 2006, \\ Australia \\ (2) Department of Interdisciplinary Studies, Faculty of Engineering, Tel Aviv University, Tel \\ Aviv 69978, Israel
}

\begin{abstract}
We introduce a model combining Kerr nonlinearity with a periodically changing sign ("nonlinearity management") and a Bragg grating (BG). The main result, obtained by means of systematic simulations, is presented in the form of a soliton's stability diagram on the parameter plane of the model; the diagram turns out to be a universal one, as it practically does not depend on the soliton's power. Moreover, simulations of the nonlinear Schrödinger (NLS) model subjected to the same "nonlinearity management" demonstrate that the same diagram determines the stability of the NLS solitons, unless they are very narrow. The stability region of very narrow NLS solitons is much smaller, and soliton splitting is readily observed in that case. The universal diagram shows that a minimum non-zero average value of Kerr coefficient is necessary for the existence of stable solitons. Interactions between identical solitons with an initial phase difference between them are simulated too in the BG model, resulting in generation of stable moving solitons. A strong spontaneous symmetry breaking is observed in the case when in-phase solitons pass through each other due to attraction between them.
\end{abstract}

PACS: 42.81.Dp, 42.65.Tg, 42.81.Qb 


\section{Introduction and formulation of the model}

Gap solitons are solitary waves in various nonlinear dispersive media, where the spectrum of linear excitations contains a gap [1]. Since solitons of this kind had been observed [2] in a short piece of a nonlinear optical fiber equipped with a Bragg grating (BG), which gives rise to the gap-bearing spectrum, they have attracted a great deal of attention in nonlinear optics. A usual model of the BG fiber is based on a system of two linearly coupled evolution equations [3, 迆:

$$
\begin{aligned}
& i u_{t}+i u_{x}+\left(\frac{1}{2}|u|^{2}+|v|^{2}\right) u+v=0 \\
& i v_{t}-i v_{x}+\left(\frac{1}{2}|v|^{2}+|u|^{2}\right) v+u=0 .
\end{aligned}
$$

Here, $u(x, t)$ and $v(x, t)$ are local amplitudes of the counterpropagating electromagnetic waves, the coefficient of the linear coupling, induced by the resonant Bragg scattering, is normalized to be 1, and the cubic terms account for the self-phase modulation (SPM) and cross-phase modulation (XPM) effects induced by the Kerr nonlinearity.

The system of Eqs. (11) and (2) is frequently called a generalized massive Thirring model. The Thirring model proper [5] corresponds to the case when the SPM terms are dropped (only in that case, the system is exactly integrable by means of the inverse scattering transform).

Although the system (11) and (2) is not integrable, it gives rise to a family of exact gapsoliton solutions [3, [4. It has been demonstrated that a part of the family is stable, and another part is unstable [6, 7]. Exact gap-soliton solutions can also be found, and their stability can be investigated, in generalized versions of the model (1), (2), which describe a system of parallelcoupled nonlinear and linear fibers with BG written on both of them [8], including also an especially interesting case when BG is written only on the linear core, so that the nonlinearity and BG are separated 9 .

There is a principal difference between the gap solitons and the commonly known solitons in ordinary single-mode nonlinear optical fibers (without BG), described by the nonlinear Schrödinger (NLS) equation 10]. In the usual NLS model, a soliton exists as a result of the balance between the self-focusing SPM nonlinearity and anomalous temporal dispersion. If the nonlinearity is self-defocusing, while the dispersion remains anomalous, bright solitons do not exist. However, in the model of the nonlinear optical fiber equipped with BG, the sign of the nonlinearity does not matter, as the effective dispersion (or diffraction, see below) induced by the grating includes both normal and anomalous branches, hence either of them will be able to support solitons. The latter circumstance suggests to consider a modified version of the standard model (1), (2) where nonlinearity's sign may change.

The simplest possibility to realize the sign-changing nonlinearity is to take it as a combination of cubic and quintic terms with opposite signs. This modification of the standard model was considered in Ref. [13]. As well as in the case of the system (11), (2), stationary soliton solutions of the modified system were found in an exact analytical form, while their stability was studied by means of numerical simulations. It has been concluded that the family of gap solitons in the modified system is drastically different from that in its standard counterpart: the family splits into two disjoint parts, each being dominated by one of the two nonlinear terms of the opposite signs (in accordance with what might be expected), and a part of each subfamily is stable.

A different possibility to study the effect of the sign-changing nonlinearity on the gap solitons is to introduce a model with the nonlinearity being represented by the cubic term only, whose sign is changing periodically as a function of the evolution variable (in analogy to dispersion management, see, e.g., Refs. [11, 12], this may be called "nonlinearity management"). In the 
assumption. However, a similar arrangement can easily be implemented in the spatial domain, i.e., for stationary light beams propagating across a layered structure in a planar nonlinear waveguide.

It is worth mentioning that stable three-wave spatial gap solitons in a model of a planar waveguide, in which BG was realized as a system of parallel scores, were found in Ref. [14. However, in that work the nonlinearity was quadratic [also known as $\chi^{(2)}$ type], and it was not subject to any modulation. On the other hand, stable $(2+1)$-dimensional (cylindrical) spatial solitons in a model of a bulk (three-dimensional) layered medium with the periodically varying sign of the cubic nonlinearity, but without BG, have recently been reported in Ref. 16] (earlier, it was demonstrated in Ref. [15] that a layered structure in the bulk medium helps to stabilize the cylindrical solitons even in the case when the size of the nonlinearity coefficient varies between the layers, without changing its sign). We do not aim to discuss in detail here how the layers with the opposite signs of the Kerr coefficient can be realized. However, it is relevant to point out that one possibility is to induce an effective Kerr coefficient via the cascading mechanism, based on the $\chi^{(2)}$ nonlinearity, where the sign of the effective $\chi^{(3)}$ coefficient can be controlled by means of the $\chi^{(2)}$ mismatch parameter [17].

Thus, the model to be considered has the form [cf. Eqs. (11) and (2)]

$$
\begin{aligned}
& i u_{z}+i u_{x}+\gamma(z)\left(\frac{1}{2}|u|^{2}+|v|^{2}\right) u+v=0, \\
& i v_{z}-i v_{x}+\gamma(z)\left(\frac{1}{2}|v|^{2}+|u|^{2}\right) v+u=0,
\end{aligned}
$$

where $z$ is the propagation distance, which plays the role of the evolution variable instead of time in Eqs. (1) and (2), and $x$ is the transverse coordinate in the layered planar waveguide. The form of Eqs. (3) and (4) implies that the carrier wave vectors of the two waves, which are resonantly reflected into each other by BG form equal angles with the $z$ axis. The reflecting scores which form the BG on the planar waveguide are oriented normally to the $z$ axis. The usual diffraction in the waveguide is neglected [similar to the negligibility of the intrinsic groupvelocity dispersion of the optical fiber in the temporal-domain model based on Eqs. (1) and (2)], as it is assumed that BG gives rise to a much stronger diffraction, cf. a similar situation in the work [14].

Although Eqs. (3) and (4) are $z$-dependent, they conserve the net power,

$$
P \equiv \int_{-\infty}^{+\infty}\left(|u(x)|^{2} d x+|v(x)|^{2}\right) d x .
$$

The equations can be represented in a Hamiltonian form, but, unlike the net power, the corresponding Hamiltonian is not a dynamical invariant, due to the presence of the explicit $z$ dependence in it.

The layered structure of the waveguide assumes that the Kerr coefficient $\gamma(z)$ takes positive and negative values $\gamma_{+}$and $\gamma_{-}$in the alternating layers (cf. Ref. [16]):

$$
\gamma(z)=\left\{\begin{array}{lr}
\gamma_{+}, & \text {if } 0<z<L_{+} \\
\gamma_{-}, & \text {if } L_{+}<z<L_{+}+L_{-}
\end{array}\right.
$$

which is repeated periodically with the period $L \equiv L_{+}+L_{-}$. Using the scaling invariance of Eqs. (3) and (4), one may always impose the following normalization conditions:

$$
L_{+}+L_{-} \equiv 1, L_{+} \gamma_{+}+L_{-}\left|\gamma_{-}\right| \equiv 1 .
$$

Thus, the model contains two irreducible control parameters, which may be selected as, e.g., $L_{+}$ and $\gamma_{+}$, while the other parameters can be found from Eqs. (尹), 
Note that corresponding average value of the Kerr coefficient is

$$
\bar{\gamma} \equiv \frac{L_{+} \gamma_{+}+L_{-} \gamma_{-}}{L_{+}+L_{-}}=2 L_{+} \gamma_{+}-1
$$

As we are interested in the sign-changing model, with $\gamma_{-}<0$, we will confine the consideration to the case $L_{+} \gamma_{+} \leq 1$, which is equivalent to $\gamma_{-} \leq 0$ according to Eqs. (8).

As is known, broad-gap-soliton solutions to Eqs. (11) and (2) are asymptotically equivalent to broad NLS solitons. This suggests to consider, parallel to the model (3), (4), also the NLS equation with the nonlinearity coefficient subjected to the same periodic modulation as in Eq. (6),

$$
i u_{z}+\frac{1}{2} u_{x x}+\gamma(z)|u|^{2} u=0
$$

where the variable $x$ may be realized as either the transverse coordinate in the planar waveguide, or the standard temporal coordinate in an optical fiber [10], $z$ being the propagation distance in either case. Comparing the results for the gap and NLS solitons in the two models will be quite helpful in realizing the generality of the conclusions presented below.

The rest of the paper is organized as follows. In section 2 we present the main results obtained in this work, in the form of a stability diagram for the solitons in the BG model, its remarkable features being that the stability region virtually does not depend on the soliton's power, and a non-zero average value of the Kerr coefficient is necessary for the existence of stable solitons. Similar results for the NLS model are presented, in a brief form (as they differ from results for the gap solitons only in the case when the solitons are very narrow), in section 3. In section 4, results produced by interactions between two stable gap solitons with a phase difference between them are presented; in particular, stable moving solitons are readily generated by the interaction. The paper is concluded by section 5 .

\section{The stability diagram for the gap solitons}

The main issue of this work is the existence of stable solitons in the model. Obviously, a soliton may only be a pulsating one ("breather"), due to the periodic inhomogeneity of the system. For the same reason, the problem is too difficult for an analytical treatment. In principle, one may try to develop an analytical approach for pulsating solitons based on the variational approximation, as it was done successfully in the case of the layered medium described by the $(2+1)$-dimensional NLS equation with the periodically changing sign of the Kerr coefficient [16]; however, unlike the NLS solitons, the variational approximation for the gap solitons is very complex even without the periodic modulation, see Ref. [6]. Therefore, we rely on direct numerical simulations of Eqs. (3) and (4), with the coefficient $\gamma(z)$ defined as per Eqs. (6) ) and (8).

At $z=0$, we used the well-known exact gap-soliton solution of the standard model (11) and (2) [3, 田:

$$
\begin{aligned}
& u_{0}(x)=(1 / \sqrt{3})(\sin \theta) \operatorname{sech}\left(x \sin \theta-\frac{1}{2} i \theta\right), \\
& v_{0}(x)=-(1 / \sqrt{3})(\sin \theta) \operatorname{sech}\left(x \sin \theta+\frac{1}{2} i \theta\right),
\end{aligned}
$$

where $\theta$ is an internal parameter of the soliton family, which takes values $0<\theta<\pi$. As is known, these solitons are stable in the interval $0<\theta<\theta_{\text {cr }}$, where the border (critical) value is slightly larger than $\left.\pi / 2, \theta_{\text {cr }} \approx 0.506 \cdot \pi[6,7]\right)$. We ran the simulations for a fixed value of the parameter $\theta$ in the initial configuration (11), while the model's control parameters $\gamma_{+}$and $L_{+}$ were gradually varied, subject to the above-mentioned constraint $L_{+} \gamma_{+} \leq 1$. Then, the same was done for other values of $\theta$. 
region shown in Fig. 1 is $L_{+} \gamma_{+}=1$, which, as was explained above, limits the case considered in this work, as the local Kerr coefficient does not change sign above this boundary. The upper boundary itself corresponds, as per Eqs. (8), to a system in which nonlinear layers of the width $L_{+}$alternate with linear ones (having $\gamma_{-}=0$ ) of the width $L_{-}$. The simulations demonstrate that everywhere on this boundary, the solitons are stable, and they remain stable above the boundary, so that it only bounds the region that we aim to consider in this work. It is noteworthy that the system composed of alternating nonlinear and linear segments is similar to the recently introduced model of a split-step fiber-optic link, which also readily gives rise to stable solitons, despite its strongly heterogeneous structure [18].

The lower boundary of the stability region in Fig. 1 is rather close to a hyperbola, the product $L_{+} \gamma_{+}$along this boundary taking values between 0.65 and 0.70 . For comparison, the dashed curve in Fig. 1 shows the hyperbola $L_{+} \gamma_{+}=1 / 2$, along which the average Kerr coefficient (9) exactly vanishes. The finite separation between the lower stability boundary and the dashed curve can be measured by the average value $\bar{\gamma}$ of the Kerr coefficient (91). The smallest value for $\bar{\gamma}$ found on the lower boundary is approximately 0.3 . Thus, stable solitons are not possible in a system where the average value of Kerr coefficient is zero. This is, incidentally, a noteworthy difference from the dispersion-managed fiber-optic systems, where stable solitons have been found in the case when the average dispersion exactly vanishes 12 .

The left vertical boundary of the stability region in Fig. 1 at $L_{+}=0.2$ is not a real stability border. It only bounds a range in which the systematic simulations were performed, which is $0.2 \leq L_{+}<1.0$.

The result described above is similar to that obtained in Ref. [16] for the (2+1)-dimensional solitons in a layered medium without BG: there too, a finite positive average value of the Kerr coefficient was necessary for the existence of any soliton, stable or unstable. It will be shown in the next section that the same result is also true for the one-dimensional layered NLS system. On the other hand, a difference of the present model from the NLS one is that the sign of the nonlinearity is not crucial (as it was explained in detail above). Indeed, the substitution $u^{*} \equiv \widetilde{u}$, $v^{*}=-\widetilde{v}$ transforms Eqs. (3) and (4) into themselves, but with the opposite sign of the Kerr coefficient $\gamma(z)$, i.e., with

$$
\gamma(z)=\left\{\begin{array}{l}
\widetilde{\gamma}_{+} \equiv\left|\gamma_{-}\right|, \quad \text { if } 0<z<\widetilde{L}_{+} \equiv L_{-}, \\
\widetilde{\gamma}_{-} \equiv-\gamma_{+}, \quad \text { if } \quad \widetilde{L}_{+}<z<L_{+}+L_{-}
\end{array} .\right.
$$

Therefore, there must exist another stability area in the region where the average Kerr coefficient is negative, i.e., $L_{+} \gamma_{+}<1 / 2$. However, the distribution ("nonlinearity-management map") of the Kerr coefficient corresponding to Eq. (12) does not satisfy the second normalization condition (7). Therefore, it is necessary to additionally redefine the wave fields, so that

$$
(\widetilde{u}, \widetilde{v}) \equiv \frac{(\widetilde{\widetilde{u}}, \widetilde{\widetilde{v}})}{\sqrt{\widetilde{L}_{+} \widetilde{\gamma}_{+}+\widetilde{L}_{-}\left|\widetilde{\gamma}_{-}\right|}}=(\widetilde{\widetilde{u}}, \widetilde{\widetilde{v}}) \sqrt{\frac{1-L_{+}}{L_{+}+\gamma_{+}-2 L_{+} \gamma_{+}}}
$$

where $\widetilde{L}_{\mp} \equiv L_{ \pm}$, and Eqs. (\$) and ( 12 ) were taken into regard. Finally, with regard to the transformation (13), the second stability region (not shown in Fig. 1), corresponding to the negative average value of the Kerr coefficient, is obtained from the region shown in Fig. 1 by the transformation

$$
\left(L_{+} ; \gamma_{+}\right) \rightarrow\left(1-L_{+} ; \frac{1-L_{+} \gamma_{+}}{L_{+}+\gamma_{+}-2 L_{+} \gamma_{+}}\right) .
$$

In the cases when the initial pulse (11) gives rise to a stable soliton, the formation of the 
conspicuous in the case when a stable soliton is in the proximity of the lower stability border. On the other hand, in the unstable case the initial pulse completely decays into radiation; a typical example is shown in Fig. 3. Strong symmetry breaking evident in Figs. 2 and 3 has, plausibly, the same reason as in Fig. 7(a), which is discussed below.

The stability diagram displayed in Fig. 1 was obtained by simulations of Eqs. (3) and (4) with the initial conditions (11) having $\theta=0.484 \cdot \pi$, which is close to the above-mentioned stabilitylimiting value $\theta_{\mathrm{cr}} \approx 0.506 \cdot \pi$ of the standard model (1), (2) which has $\gamma \equiv 1$. The stability region may, of course, depend on the initial value of $\theta$, or, in other words, on the initial value of the soliton's power, defined by the expression (5), which is $P_{\text {sol }}=(4 / 3) \theta$ for the configuration (11). As for the dependence of the stability region [defined in terms of the parameter plane $\left(L_{+}, \gamma_{+}\right)$, as in Fig. 1 ] on soliton's power, one may naturally expect that the dependence is strongest when the initial value of $\theta$ is close to the above-mentioned value $\theta_{\text {cr }}$, which is the stability border for solitons in the ordinary BG model (11), (2). Our simulations show that, in fact, the stability region shows virtually no sensitivity to the variation of $\theta$ : for example, decreasing $\theta$ from the value $\theta=\cos ^{-1}(0.05) \approx 0.484 \cdot \pi$, corresponding to Fig. 1 , to $\theta=\cos ^{-1}(0.15) \approx 0.452 \cdot \pi$, produces no visible change whatsoever in the shape of the stability region. Because the region shows no sensitivity to the initial power in the case when the sensitivity might be strongest, we conjecture (which is also corroborated by additional simulations for essentially smaller values of $\theta$ ) that the stability diagram shown in Fig. 1 is a universal one, without any tangible dependence upon the soliton's power.

In this connection, it may be relevant to mention a somewhat similar stability property of the gap solitons in the ordinary model (11) and (2): besides the zero-velocity ones given by Eqs. (11), more general analytical solutions for solitons moving at an arbitrary velocity $V$, which takes values in the interval $-1<-V<1$, are also known [3, 14. This generalization of the exact soliton solutions is not trivial, as Eqs. (11) and (2) do not feature any invariance with respect to the change of the reference frame; for the same reason, the stability limit for the moving solitons cannot be obtained by any straightforward transformation from that for the zerovelocity ones. Nevertheless, direct numerical results [7] show that, although the corresponding limit value $\theta_{\text {cr }}$ does depend on $V$, the dependence is very weak: as $V$ increases from 0 to 1 , the stability boundary gradually moves from the above-mentioned value $\theta_{\mathrm{cr}}(V=0) \approx 0.506 \cdot \pi$ to $\theta_{\mathrm{cr}}(V=1) \approx 0.508 \cdot \pi$.

\section{Stability of solitons in the NLS equation with spatially mod- ulated nonlinearity}

It is well known that the BG model based on Eqs. (11) and (2) is related to the NLS equation: in the limit $\theta \rightarrow 0$, a small-amplitude broad gap soliton given by Eqs. (11) is asymptotically equivalent to the NLS soliton. Therefore, in the limit of small $\theta$ Eqs. (3) and (14) are tantamount to the NLS equation (10) with the periodically modulated nonlinear coefficient. On the other hand, for solitons with a small amplitude $\theta$ and a large width $\sim 1 / \theta$, a characteristic diffraction length, which determines the dynamical scale for the evolution of the soliton, is $\sim 1 / \theta^{2}$. This length is much larger than the modulation period of the Kerr coefficient, which is $L \equiv 1$ in the present notation, hence the small-amplitude broad soliton will feel only the average value (9) of the Kerr coefficient. This, in turn, means that the broad solitons should be stable in both the BG and NLS models for any positive value of the average Kerr coefficient $\bar{\gamma}$.

The latter conclusion appears to be in contradiction with the results presented in Fig. 1, where, as was stressed above, for all the stable solitons the average Kerr coefficient $\bar{\gamma}$ was found to exceed the minimum value $\approx 0.3$. However, direct simulations of very broad solitons in either 
results, as very long propagation is necessary, in view of the large value of the above-mentioned diffraction length for these solitons. Besides that, it is not easy to distinguish a small-amplitude soliton from radiation waves which it sheds off in the course of the evolution. Lastly, this issue, i.e., finding exact stability limits for very broad gap and NLS solitons, is of no practical interest, as experiments with such solitons would require very large samples of the layered waveguide and would therefore be extremely difficult, not promising anything for applications. Therefore, we did not try to investigate this issue in detail.

Nevertheless, for the sake of the comparison between the two models just in the case when they are really different, it is of interest to generate a stability diagram for (not-very-broad) NLS solitons, of the same type as the diagram displayed in Fig. 1. To this end, systematic simulations of Eq. (10) were performed, with $\gamma(z)$ taken as per Eqs. (6), ( 8). The simulations began with the initial condition

$$
u_{0}(x)=\eta \operatorname{sech}(\eta x),
$$

that would generate an exact soliton in the NLS equation with $\gamma \equiv 1$. In view of the abovementioned difficulties with simulations of very broad solitons, definite results were obtained for the initial configurations (14) with $\eta \geq \eta_{\min }=0.5$ [for $\eta=0.4$, the situation is very similar, but residual evolution of the soliton does not completely cease during the simulation time; note the $\eta=0.5$ corresponds, roughly, to $\theta=\pi / 6$ in the case of the gap soliton (11), if the comparison is made by the soliton's width] .

The result is that, for all the moderately narrow initial solitons of the type (14), the thus obtained stability diagram is virtually indistinguishable from its counterpart in Fig. 1. This finding once again stresses the universal character of the stability diagram presented in Fig. 1, as it applies to both models in the case when they are really different.

The difference between the BG and NLS models becomes drastic if the amplitude $\eta$ of the initial pulse (14) is high (recall that the amplitude of the NLS soliton may be indefinitely large, while the amplitude of the gap solitons (11), which is $(2 / \sqrt{3}) \sin (\theta / 2)$, does not exceed the maximum value $2 / \sqrt{3}$ ). It is obvious that, for very narrow solitons with large $\eta$, whose diffraction length $\sim 1 / \eta^{2}$ is much smaller than the modulation period $L=1$, the periodic change of the nonlinearity sign, as per Eq. (6), is a strong perturbation that may destroy the soliton. Indeed, running the simulations for the NLS equation (10) with the initial condition (10), we have observed that the stability region strongly shrinks (compared with that shown in Fig. 1) in the case of large $\eta$. Moreover, even if the evolution of the initial pulse (14) with large $\eta$ results in the appearance of stable solitons, they were frequently produced by splitting of the initial pulse. The situation was classified as a stable one if stable solitons finally appeared in any fashion.

An example of the thus defined stability diagram for the NLS model (10) with a large initial amplitude $\eta=5$ is displayed in Fig. 4, and an example of the splitting of the initial pulse into two secondary solitons with a much smaller amplitude is presented in Fig. 5. In the case $\eta=5$, the splitting was always observed if, for instance, the parameter $L_{+}$was fixed to be 0.4 . We did not aim to find the smallest value of the initial amplitude which gives rise to the splitting, as this would require running extremely long simulations (it is plausible that the splitting distance diverges around that smallest value of $\eta$ ).

In some other cases, a stable soliton, with an amplitude essentially smaller than the large initial amplitude, appears as a result of what may be interpreted as splitting of the initial pulse into a central soliton and two very weak additional solitons which move aside. A typical example of this outcome is shown in Fig. 6 . 


\section{Interactions between solitons and generation of moving soli- tons}

Coming back to gap solitons, we note that, as the standard model (1), (2) is not integrable, interactions between the gap solitons are not elastic. This has already been demonstrated in the first direct simulations of the model [3]. One may, therefore, expect that interactions between stable gap solitons in Eqs. (3) and (田) will not be elastic either.

We studied interactions between gap solitons, taking two identical ones, giving them an initial phase difference $\Delta \phi$, and placing them at some distance $\Delta x$ from each other. As a result, we observed that, quite naturally, the in-phase solitons with $\Delta \phi=0$ attract each other, while out-of-phase ones with $\Delta \phi=\pi$ mutually repel, see Figs. 7(a) and 7(c). In the intermediate case $\Delta \phi=\pi / 2$, the solitons also repel each other, see Fig. 7(b).

Fig. 7 demonstrates two other important features. First, it clearly shows that stable moving solitons also exists in the present model, and they can be easily generated. Second, in the case when the two solitons initially attract each other, and hence temporarily merge into a "lump" [see Fig. 7(a)], conspicuous spontaneous symmetry breaking is observed, and the outcome of the interaction is inelastic: generation of an extra moving (quasi-)soliton, along with some radiation, is clearly seen. In fact, this spontaneous symmetry breaking, in the case of the initially attractive interaction, is similar to an effect recently reported in Ref. [9]. It seems plausible that an explanation which was proposed in that paper applies to the present case as well: the above-mentioned "lump" is subject to modulational instability [10], hence the amplification of small random numerical perturbations by the instability gives rise to the symmetry breaking.

Collisions between moving solitons may also lead to inelastic interactions, but detailed simulations of such collisions are beyond the scope of this paper.

\section{Conclusion}

In this work, we have introduced a model that describes a planar nonlinear waveguide, equipped with a Bragg grating and composed of periodically alternating self-focusing and self-defocusing layers. The main result, obtained by means of systematic simulations of the evolution of solitons in the model, was presented as the soliton's stability diagram on the parameter plane of the model; it has been found that the diagram practically does not depend on the soliton's power. An important feature revealed by the stability diagram is that a minimum non-zero value of the average Kerr coefficient is necessary for the existence of stable solitons.

To compare the result with the nonlinear Schrödinger model, where the Kerr coefficient is subjected to the same sign-changing modulation as in the Bragg-grating model, the NLS model was also simulated in a systematic way. It has been found that, for moderately narrow NLS solitons, the stability diagram is virtually identical to that for the gap solitons, which stresses the universal character of this diagram. For very narrow NLS solitons, the stability region is much smaller, and, in the latter case, stable solitons frequently appear as a result of splitting of the initial pulse.

Interactions between two identical stable gap solitons with an initial phase difference between them were simulated too. As a result, stable moving solitons were easily generated, and a strong spontaneous symmetry breaking was observed in the case when two in-phase solitons pass through each other. 


\section{References}

[1] C.M. de Sterke and J.E. Sipe, in Progress in Optics, edited by E. Wolf (Elsevier, NorthHolland, 1994), Vol. XXXIII, Chap. 3, p. 205.

[2] B.J. Eggleton, R.E. Slusher, C.M. de Sterke, P.A. Krug, and J.E. Sipe, Phys. Rev. Lett. 76 (1996) 1627; C.M. de Sterke, B.J. Eggleton, and P.A. Krug, J. Lightwave Technol. 15 (1997) 1494; N.G.R. Broderick, D.J. Richardson, R.I. Laming, and M. Ibsen, Opt. Lett. 23 (1998) 328 (1998).

[3] A.B. Aceves and S. Wabnitz, Phys. Lett. A 141 (1989) 37.

[4] D.N. Christodoulides and R.I. Joseph, Phys. Rev. Lett. 62 (1989) 1746.

[5] W.E. Thirring, Ann. Phys. (NY) 3 (1958) 91.

[6] B.A. Malomed and R.S. Tasgal, Phys. Rev. E 49 (1994) 5787.

[7] I.V. Barashenkov, D.E. Pelinovsky, and E.V. Zemlyanaya, Phys. Rev. Lett. 80 (1998) 5117.

[8] J. Atai and B.A. Malomed, Phys. Rev. E 62 (2000) 8713.

[9] J. Atai and B.A. Malomed, Phys. Rev. E 64 (2001), paper No. 06617.

[10] G.P. Agrawal. Nonlinear Fiber Optics (Academic Press: Boston, 1995).

[11] J.H.B. Nijhof, N.J. Doran, W. Forysiak and F.M. Knox, Electron. Lett. 33 (1997) 1726.

[12] A. Berntson, N.J. Doran, W. Forysiak, and J.H.B. Nijhof, Opt. Lett. 23 (1998) 900; S.K. Turitsyn and E.G. Shapiro, Opt. Lett. 23 (1998) 682; T.I. Lakoba, J. Yang, D.K. Kaup, and B.A. Malomed, Opt. Commun. 149 (1998) 366.

[13] J. Atai and B.A. Malomed, Phys. Lett. A 284 (2001) 247.

[14] W.C.K. Mak, B.A. Malomed, and P.L. Chu, Phys. Rev. E 58 (1998) 6708.

[15] L. Bergé, V.K. Mezentsev, J. Juul Rasmussen, P.L. Christiansen, and Yu.B. Gaididei, Opt. Lett. 25 (2000) 1037.

[16] I. Towers and B.A. Malomed, J. Opt. Soc. Am. B, in press.

[17] L.J. Qian, X. Liu, and F.W. Wise, Opt. Lett. 24 (1999) 166; X. Liu, L. Qian, and F. Wise, Opt. Lett. 24 (1999) 1777.

[18] R. Driben and B.A. Malomed, Opt. Commun. 185 (2000) 439. 


\section{Figure Captions}

Fig. 1. The stability diagram for solitons in the model based on Eqs. (3), (4) and (6), (8). The stability region is bounded by the lower solid curve, while the upper curve, the hyperbola $L_{+} \gamma_{+}=1$, is a border of the parametric area where the local Kerr coefficient periodically changes its sign. The dashed curve is the hyperbola $L_{+} \gamma_{+}=1 / 2$ along which the average value of nonlinearity is zero.

Fig. 2. An example of the formation of a stable soliton when $L_{+}=0.5$ and $\gamma_{+}=1.7$. Only the $u$ component is shown.

Fig. 3. An example of the decay of the initial pulse (11) into radiation at a point belonging to the unstable region in Fig. 1 , with $L_{+}=0.5$ and $\gamma_{+}=1.2$. Only the $u$ component is shown.

Fig. 4. A narrow stability region in the nonlinear Schrödinger model (10) where the Kerr coefficient periodically changes its sign as per Eqs. (6) and (8) for the case when the initial pulse is given by Eq. (14) with $\eta=5$.

Fig. 5. An example of splitting of the narrow pulse in the NLS model (10), (14) into two secondary solitons with a smaller amplitude in the case $\eta=5$. The parameters of the model are $L_{+}=0.35$ and $\gamma_{+}=2.74$. The propagation distance shown in this figure is 200 (i.e., 200 modulation periods).

Fig. 6. An example of spitting of the narrow pulse in the NLS model (10), (14) into a central soliton with a smaller amplitude and two very weak side pulses in the case $\eta=5$. The parameters of the model are almost the same as in Fig. 5, with a difference that $\gamma_{+}=2.76$.

Fig. 7. The interaction of two identical stable solitons, with the initial phase difference $\Delta \phi$ and separation $\Delta x$ between their centers, when $L_{+}=0.5, \gamma_{+}=1.94$. The solitons have been generated from the initial configuration (11) with $\theta \approx 0.484 \cdot \pi$ (the same value as the one used in Fig. 1). The three cases displayed in the figure correspond to (a) $\Delta \phi=0, \Delta x=12$; (b) $\Delta \phi=\pi / 2, \Delta x=8$; (c) $\Delta \phi=\pi, \Delta x=12$. Only the u component is shown. 